\title{
Inverse Scattering Transform for the Camassa-Holm equation
}

\author{
Adrian Constantin ${ }^{a, \dagger}$, Vladimir S. Gerdjikov ${ }^{b, \ddagger}$ and \\ Rossen I. Ivanov ${ }^{a, *, 1}$
}

\author{
${ }^{a}$ School of Mathematics, Trinity College Dublin, \\ Dublin 2, Ireland \\ ${ }^{b}$ Institute for Nuclear Research and Nuclear Energy, \\ Bulgarian Academy of Sciences, \\ 72 Tzarigradsko chaussee, 1784 Sofia, Bulgaria \\ ${ }^{\dagger}$ e-mail: adrian@maths.tcd.ie \\ ‡e-mail: gerjikov@inrne.bas.bg \\ *e-mail:ivanovr@tcd.ie
}

\begin{abstract}
An Inverse Scattering Method is developed for the Camassa-Holm equation. As an illustration of our approach the solutions corresponding to the reflectionless potentials are constructed in terms of the scattering data. The main difference with respect to the standard Inverse Scattering Transform lies in the fact that we have a weighted spectral problem. We therefore have to develop different asymptotic expansions.
\end{abstract}

MSC: 35P25, 35Q15, 35Q35, 35Q51, 35Q53

Key Words: Hamiltonian systems, Integrable Systems, Lax Pair, Riemann-Hilbert Problem, Solitons.

\section{Introduction}

In this introductory section some well known facts about the Camassa-Holm $(\mathrm{CH})$ equation and the related spectral problem will be highlighted. The $\mathrm{CH}$ equation [5]

$$
u_{t}-u_{x x t}+2 \omega u_{x}+3 u u_{x}-2 u_{x} u_{x x}-u u_{x x x}=0,
$$

where $\omega$ is a real constant, gained popularity as a model describing the unidirectional propagation of shallow water waves over a flat bottom [5, 25.

\footnotetext{
${ }^{1}$ On leave from the Institute for Nuclear Research and Nuclear Energy, Bulgarian Academy of Sciences, Sofia, Bulgaria.
} 
27. as well as that of axially symmetric waves in a hyperelastic rod [16, 18]. It firstly appeared in [20] as an equation with a bi-hamiltonian structure. $\mathrm{CH}$ is a completely integrable equation [5, 6, 1, 14, 9, 29, 21, describing permanent and breaking waves [10, 8]. Its solitary waves are stable solitons if $\omega>0$ 2, 15, 17, 26 or peakons if $\omega=0,5,6$. CH arises also as an equation of the geodesic flow for the $H^{1}$ right-invariant metrics on the Bott-Virasoro group (if $\omega>0$ ) and on the diffeomorphism group (if $\omega=0$ ) [34, 13, 8, 12].

The bi-Hamiltonian form of (11) is [5, 20]:

$$
m_{t}=-\left(\partial-\partial^{3}\right) \frac{\delta H_{2}[m]}{\delta m}=-(2 \omega \partial+m \partial+\partial m) \frac{\delta H_{1}[m]}{\delta m} .
$$

where

$$
m=u-u_{x x}
$$

and the Hamiltonians are

$$
\begin{aligned}
H_{1}[m] & =\frac{1}{2} \int m u d x \\
H_{2}[m] & =\frac{1}{2} \int\left(u^{3}+u u_{x}^{2}+2 \omega u^{2}\right) d x .
\end{aligned}
$$

The integration is from $-\infty$ to $\infty$ in the case of Schwartz class functions, and over one period in the periodic case.

There exists an infinite sequence of conservation laws (multi-Hamiltonian structure) $H_{n}[m], n=0, \pm 1, \pm 2, \ldots$, such that [19, 40, 30, 24]

$$
-\left(\partial-\partial^{3}\right) \frac{\delta H_{n}[m]}{\delta m}=-(2 \omega \partial+m \partial+\partial m) \frac{\delta H_{n-1}[m]}{\delta m} .
$$

The equation (11) admits a Lax pair [5, 9]

$$
\begin{aligned}
\Psi_{x x} & =\left(\frac{1}{4}+\lambda(m+\omega)\right) \Psi \\
\Psi_{t} & =\left(\frac{1}{2 \lambda}-u\right) \Psi_{x}+\frac{u_{x}}{2} \Psi+\gamma \Psi
\end{aligned}
$$

where $\gamma$ is an arbitrary constant. We will use this freedom for a proper normalization of the eigenfunctions.

In our further considerations $m$ will be a Schwartz class function, $\omega>0$ and $m(x, 0)+\omega>0$. Then $m(x, t)+\omega>0$ for all $t[9$. For a discussion of the periodic case we refer to [14] and [7]. Let $k^{2}=-\frac{1}{4}-\lambda \omega$, i.e.

$$
\lambda(k)=-\frac{1}{\omega}\left(k^{2}+\frac{1}{4}\right) .
$$


The spectrum of the problem (7) under these conditions is described in 9]. The continuous spectrum in terms of $k$ corresponds to $k$-real. The discrete spectrum (in the upper half plane) consists of finitely many points $k_{n}=i \kappa_{n}, n=1, \ldots, N$ where $\kappa_{n}$ is real and $0<\kappa_{n}<1 / 2$.

For all real $k \neq 0$ a basis in the space of solutions of (17) can be introduced, fixed by its asymptotic when $x \rightarrow \infty[9]$ :

$$
\begin{aligned}
& \psi_{1}(x, k)=e^{-i k x}+o(1), \quad x \rightarrow \infty \\
& \psi_{2}(x, k)=e^{i k x}+o(1), \quad x \rightarrow \infty
\end{aligned}
$$

Another basis can be introduced, fixed by its asymptotic when $x \rightarrow-\infty$ :

$$
\begin{aligned}
& \varphi_{1}(x, k)=e^{-i k x}+o(1), \quad x \rightarrow-\infty \\
& \varphi_{2}(x, k)=e^{i k x}+o(1), \quad x \rightarrow-\infty .
\end{aligned}
$$

For all real $k \neq 0$ if $\Psi(x, k)$ is a solution of (7), then $\Psi(x,-k)$ is also a solution, thus

$$
\varphi_{1}(x, k)=\varphi_{2}(x,-k), \quad \psi_{1}(x, k)=\psi_{2}(x,-k) .
$$

Due to the reality of $m$ in (17) for any $k$ we have

$$
\varphi_{1}(x, k)=\bar{\varphi}_{2}(x, \bar{k}), \quad \psi_{1}(x, k)=\bar{\psi}_{2}(x, \bar{k})
$$

The vectors of each of the bases are a linear combination of the vectors of the other basis:

$$
\varphi_{i}(x, k)=\sum_{l=1,2} T_{i l}(k) \psi_{l}(x, k)
$$

where the matrix $T(k)$ defined above is called the scattering matrix. For real $k \neq 0$, instead of $\varphi_{1}(x, k), \varphi_{2}(x, k), \psi_{1}(x, k), \psi_{2}(x, k)$ due to (15), for simplicity we can write correspondingly $\varphi(x, k), \bar{\varphi}(x, k), \psi(x, k), \bar{\psi}(x, k)$. Thus $T(k)$ has the form

$$
T(k)=\left(\begin{array}{cc}
a(k) & b(k) \\
\bar{b}(k) & \bar{a}(k)
\end{array}\right)
$$

and clearly

$$
\varphi(x, k)=a(k) \psi(x, k)+b(k) \bar{\psi}(x, k) .
$$


The Wronskian $W\left(f_{1}, f_{2}\right) \equiv f_{1} \partial_{x} f_{2}-f_{2} \partial_{x} f_{1}$ of any pair of solutions of (17) does not depend on $x$. Therefore

$$
W(\varphi(x, k), \bar{\varphi}(x, k))=W(\psi(x, k), \bar{\psi}(x, k))=2 i k
$$

From (18) and (19) it follows that

$$
|a(k)|^{2}-|b(k)|^{2}=1,
$$

i.e. $\operatorname{det}(T(k))=1$.

In analogy with the spectral problem for the $\mathrm{KdV}$ equation [35, one can see that the quantities $\mathcal{T}(k)=a^{-1}(k)$ and $\mathcal{R}(k)=b(k) / a(k)$ represent themselves the transmission and reflection coefficients respectively [9, 11]. From (20) it follows that the scattering is unitary, i.e.

$$
|\mathcal{T}(k)|^{2}+|\mathcal{R}(k)|^{2}=1
$$

The entire information about $T(k)$ (17) is provided by $\mathcal{R}(k)$ for $k>0$ only [11. It is sufficient to know $\mathcal{R}(k)$ only on the half line $k>0$, since from (14) and (18), $\bar{a}(k)=a(-k), \bar{b}(k)=b(-k)$ and thus $\mathcal{R}(-k)=\overline{\mathcal{R}}(k)$.

At the points of the discrete spectrum, $a(k)$ has simple zeroes [9], therefore $\varphi$ and $\bar{\psi}$ are linearly dependent (18):

$$
\varphi\left(x, i \kappa_{n}\right)=b_{n} \bar{\psi}\left(x,-i \kappa_{n}\right) .
$$

In other words, the discrete spectrum is simple, there is only one (real) eigenfunction $\varphi^{(n)}(x)$, corresponding to each eigenvalue $i \kappa_{n}$, and we can take this eigenfunction to be

$$
\varphi^{(n)}(x) \equiv \varphi\left(x, i \kappa_{n}\right)
$$

The asymptotic of $\varphi^{(n)}$, according to (12), (11), (22) is

$$
\begin{aligned}
& \varphi^{(n)}(x)=e^{\kappa_{n} x}+o\left(e^{\kappa_{n} x}\right), \quad x \rightarrow-\infty ; \\
& \varphi^{(n)}(x)=b_{n} e^{-\kappa_{n} x}+o\left(e^{-\kappa_{n} x}\right), \quad x \rightarrow \infty .
\end{aligned}
$$

The sign of $b_{n}$ obviously depends on the number of the zeroes of $\varphi^{(n)}$. Suppose that

$$
0<\kappa_{1}<\kappa_{2}<\ldots<\kappa_{N}<1 / 2 .
$$


Then from the oscillation theorem for the Sturm-Liouville problem [4], $\varphi^{(n)}$ has exactly $n-1$ zeroes. Therefore

$$
b_{n}=(-1)^{n-1}\left|b_{n}\right|
$$

The set

$$
\mathcal{S} \equiv\left\{\mathcal{R}(k) \quad(k>0), \quad \kappa_{n}, \quad\left|b_{n}\right|, \quad n=1, \ldots N\right\}
$$

is called scattering data. The Hamiltonians for the $\mathrm{CH}$ equation in terms of the scattering data are presented in [11.

The time evolution of the scattering data can be easily obtained as follows. From (18) with $x \rightarrow \infty$ one has

$$
\varphi(x, k)=a(k) e^{-i k x}+b(k) e^{i k x}+o(1) .
$$

The substitution of $\varphi(x, k)$ into (8) with $x \rightarrow \infty$ gives

$$
\varphi_{t}=\frac{1}{2 \lambda} \varphi_{x}+\gamma \varphi
$$

From (29), (30) with the choice $\gamma=i k / 2 \lambda$ for the eigenfunction $\varphi(x, k)$ we obtain

$$
\begin{aligned}
\dot{a}(k, t) & =0, \\
\dot{b}(k, t) & =\frac{i k}{\lambda} b(k, t),
\end{aligned}
$$

where the dot stands for derivative with respect to $t$. Thus

$$
\begin{gathered}
a(k, t)=a(k, 0), \quad b(k, t)=b(k, 0) e^{\frac{i k}{\lambda} t} \\
\mathcal{T}(k, t)=\mathcal{T}(k, 0), \quad \mathcal{R}(k, t)=\mathcal{R}(k, 0) e^{\frac{i k}{\lambda} t} .
\end{gathered}
$$

In other words, $a(k)$ is independent on $t$ and will serve as a generating function of the conservation laws.

The time evolution of the data on the discrete spectrum is found as follows. $i \kappa_{n}$ are zeroes of $a(k)$, which does not depend on $t$, and therefore $\dot{\kappa}_{n}=0$. From (8) with $\gamma=i k / 2 \lambda ; k=i \kappa_{n}$ and (25) one can obtain

$$
\dot{b}_{n}=\frac{4 \omega \kappa_{n}}{1-4 \kappa_{n}^{2}} b_{n} .
$$


The Poisson brackets for the scattering data of the Camassa-Holm equation are computed in [11] where also the action-angle variables are expressed in terms of the scattering data.

In Section 2 we compute the asymptotics for large $k$ of the scattering data and the eigenfunctions, which we use in Section 3 to develop the Inverse Scattering Transform for the $\mathrm{CH}$ equation. A number of recent papers [9, 26, 31, 32] used a Liouville transformation to reduce the weighted spectral problem (77) to a standard problem. Our approach is more direct, and provides, we believe, more transparent formulas. The special case of reflectionless potentials $(\mathcal{R}(k)=0$ for all $k$ ) which corresponds to the important class of solutions, namely the multi-soliton solutions is separately studied in Section 4. A formula for the $N$-soliton solution is obtained.

\section{Analytic solutions and Riemann-Hilbert Prob- lem}

For the application of the Inverse Scattering Method it will be necessary the asymptotics for large $k$ of $a(k)$ and the Jost solutions to be found. Firstly we compute the asymptotic of $a(k)$.

The solution of (7) can be represented in the form

$$
\varphi(x, k)=\exp \left(-i k x+\int_{-\infty}^{x} \chi(y, k) d y\right) .
$$

For $\operatorname{Im} k>0$ and $x \rightarrow \infty, \varphi(x, k) e^{i k x}=a(k)$, i.e.

$$
\ln a(k)=\int_{-\infty}^{\infty} \chi(x, k) d x, \quad \operatorname{Im} k>0 .
$$

Since $a(k)$ does not depend on $t$, the expressions $\int_{-\infty}^{\infty} \chi(x, k) d x$ represent integrals of motion for all $k$. The equation for $\chi(x, k)$ follows from (7) and (36)

$$
\chi_{x}(x, k)+\chi^{2}-2 i k \chi=-\frac{1}{\omega}\left(k^{2}+\frac{1}{4}\right) m(x)
$$

and admits a solution with the asymptotic expansion

$$
\chi(x, k)=p_{1} k+p_{0}+\sum_{n=1}^{\infty} \frac{p_{-n}}{k^{n}} .
$$

The substitution of (39) into (38) gives the following quadratic equation for $p_{1}$ :

$$
p_{1}^{2}-2 i p_{1}+\frac{m}{\omega}=0
$$


with solutions

$$
p_{1}=i\left(1 \pm \sqrt{1+\frac{m}{\omega}}\right)
$$

Since $\int_{-\infty}^{\infty} p_{1}(x) d x$ is an integral of the $\mathrm{CH}$ equation, presumably finite, we take the minus sign in (41). One can easily see that $p_{0}$ and all $p_{-2 n}$ are total derivatives [24] and thus we have the expansion

$$
\ln a(k)=-i \alpha k+\sum_{n=1}^{\infty} \frac{I_{-n}}{k^{n}}
$$

where $\alpha$ is a positive constant (integral of motion):

$$
\alpha=\int_{-\infty}^{\infty}\left(\sqrt{1+\frac{m(x)}{\omega}}-1\right) d x
$$

and $I_{-n}=\int_{-\infty}^{\infty} p_{-n}$ are the other integrals, whose densities, $p_{-n}$ can be obtained reccurently from (38), (39) [24, 11].

In terms of the scattering data $\alpha$ can be expressed as [11]

$$
\alpha=\sum_{n=1}^{N} \ln \left(\frac{1+2 \kappa_{n}}{1-2 \kappa_{n}}\right)^{2}-\frac{8}{\pi} \int_{0}^{\infty} \frac{\ln |a(\widetilde{k})|}{4 \widetilde{k}^{2}+1} d \widetilde{k} .
$$

The asymptotic of $a(k)$ for $\operatorname{Im} k>0$ and $|k| \rightarrow \infty$ from (42) is $a(k) \rightarrow$ $e^{-i \alpha k}$, or

$$
e^{i \alpha k} a(k) \rightarrow 1, \quad \operatorname{Im} k>0, \quad|k| \rightarrow \infty .
$$

When $k$ is in the upper half plane the following expression is valid [1]

$$
\ln a(k)=-i \alpha k+\sum_{n=1}^{N} \ln \frac{k-i \kappa_{n}}{k+i \kappa_{n}}+\frac{1}{\pi i} \int_{-\infty}^{\infty} \frac{\ln \left|a\left(k^{\prime}\right)\right|}{k^{\prime}-k} d k^{\prime} .
$$

Let us now consider the asymptotic of the Jost solutions, starting for example from $\psi(x, k)$, (10). One can check that the asymtotic for $|k| \rightarrow \infty$ has the form

$$
\begin{aligned}
& \psi(x, k)=e^{-i k x+k G(x)} \eta(x, k) \\
& \eta(x, k)=X_{0}(x)+\frac{X_{1}(x)}{k}+\frac{X_{2}(x)}{k^{2}}+\ldots,
\end{aligned}
$$

where, due to (10), $G(x) \rightarrow 0$ and $\eta(x, k) \rightarrow 1$ for $x \rightarrow \infty$. The substitution of (47) into (7) gives explicitly $G(x), X_{0}, X_{1}, \ldots$ :

$$
\psi(x, k)=e^{-i k x+i k \int_{\infty}^{x}\left(1-\sqrt{\frac{m(y)+\omega}{\omega}}\right) d y}\left[\left(\frac{\omega}{m(x)+\omega}\right)^{1 / 4}+\frac{X_{1}(x)}{k}+\ldots\right] .
$$


Introducing the function

$$
\xi(x)=\exp \left[x+\int_{\infty}^{x}\left(\sqrt{\frac{m(y)+\omega}{\omega}}-1\right) d y\right],
$$

which looks like a deformation of the ordinary exponent, (48) can be written as

$$
\psi(x, k)=[\xi(x)]^{-i k}\left[\left(\frac{\xi(x)}{\xi^{\prime}(x)}\right)^{1 / 2}+\frac{X_{1}(x)}{k}+\frac{X_{2}(x)}{k^{2}}+\ldots\right] .
$$

Furthermore, the function $\underline{\chi}(x, k) \equiv \psi(x, k) e^{i k x}$ is analytic for $\operatorname{Im} k<0$, 9]. This follows from the representation

$$
\underline{\chi}(x, k)=1-\frac{\lambda}{k} \int_{x}^{\infty} \frac{e^{2 i k\left(x-x^{\prime}\right)}-1}{2 i} m\left(x^{\prime}\right) \underline{\chi}\left(x^{\prime}, k\right) d x^{\prime} .
$$

Notice that $\int_{\infty}^{x}\left(\sqrt{\frac{m(y)+\omega}{\omega}}-1\right) d y$ is bounded for all values of $x$. Indeed,

$$
\left|\int_{\infty}^{x}\left(\sqrt{\frac{m(y)+\omega}{\omega}}-1\right) d y\right|=\left|\int_{x}^{\infty} \frac{m(y) d y}{\omega\left(1+\sqrt{\frac{m(y)+\omega}{\omega}}\right)}\right| \leq \int_{-\infty}^{\infty} \frac{|m(y)|}{\omega} d y<\infty
$$

since $m(x)$ is a Schwartz class function. Therefore the function

$$
\underline{\psi}(x, k) \equiv \psi(x, k)[\xi(x)]^{i k}
$$

is also analytic for $\operatorname{Im} k<0$.

Similarly,

$$
\begin{aligned}
\underline{\varphi}(x, k) & \equiv \varphi(x, k) \exp \left\{i k\left[x+\int_{-\infty}^{x}\left(\sqrt{\frac{m(y)+\omega}{\omega}}-1\right) d y\right]\right\} \\
& =\left(\frac{\xi(x)}{\xi^{\prime}(x)}\right)^{1 / 2}+\frac{\widetilde{X}_{1}(x)}{k}+\frac{\widetilde{X}_{2}(x)}{k^{2}}+\ldots
\end{aligned}
$$

is analytic for $\operatorname{Im} k>0$.

Multiplying (18) by $\xi(x) / a(k)$ and using (43), (52) and (53) we obtain

$$
\frac{\underline{\varphi}(x, k)}{e^{i k \alpha} a(k)}=\underline{\psi}(x, k)+\mathcal{R}(k) \underline{\bar{\psi}}(x, k)[\xi(x)]^{2 i k} .
$$

The function $\underline{\varphi}(x, k) /\left(e^{i k \alpha} a(k)\right)$ is analytic for $\operatorname{Im} k>0, \underline{\psi}(x, k)$ is analytic for $\operatorname{Im} k<0$. Thus, (54) represents an additive Riemann-Hilbert Problem with a jump on the real line, given by $\mathcal{R}(k) \underline{\bar{\psi}}(x, k)[\xi(x)]^{2 i k}$. 
Figure 1: The contours $\Gamma_{ \pm}$

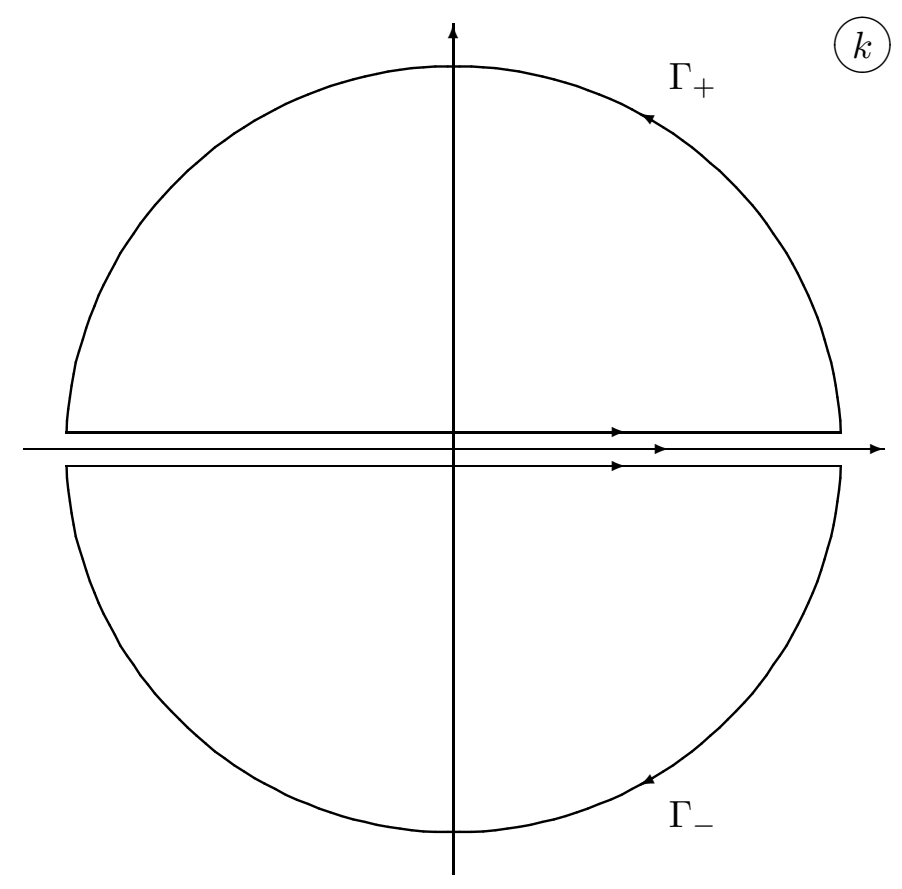

\section{Integration of the $\mathrm{CH}$ equation by the Inverse Scattering Method}

Let us take an arbitrary $k$ from the lower half plane (Im $k<0)$. Then using the Residue Theorem, (43) and (22) we can compute the integral

$$
\begin{aligned}
\frac{1}{2 \pi i} \oint_{C_{+}} \frac{\underline{\varphi}\left(x, k^{\prime}\right)}{e^{i k^{\prime} \alpha} a\left(k^{\prime}\right)} \frac{d k^{\prime}}{k^{\prime}-k} & =\sum_{n=1}^{N} \frac{\underline{\varphi}\left(x, i \kappa_{n}\right)}{\left(i \kappa_{n}-k\right) e^{-\kappa_{n} \alpha} a^{\prime}\left(i \kappa_{n}\right)} \\
& =\sum_{n=1}^{N} \frac{b_{n}[\xi(x)]^{-2 \kappa_{n}} \underline{\psi}\left(x,-i \kappa_{n}\right)}{a^{\prime}\left(i \kappa_{n}\right)\left(i \kappa_{n}-k\right)}
\end{aligned}
$$

where $C_{+}$is the closed contour in the upper half plane (Fig.1). On the other hand, due to (154) the same integral can be computed directly as 


$$
\begin{aligned}
& \frac{1}{2 \pi i} \oint_{C_{+}} \frac{\underline{\varphi}\left(x, k^{\prime}\right)}{e^{i k^{\prime} \alpha} a\left(k^{\prime}\right)} \frac{d k^{\prime}}{k^{\prime}-k} \\
&=\frac{1}{2 \pi i} \int_{-\infty}^{\infty}\left(\underline{\psi}\left(x, k^{\prime}\right)+\mathcal{R}\left(k^{\prime}\right) \underline{\bar{\psi}}\left(x, k^{\prime}\right)[\xi(x)]^{2 i k^{\prime}}\right) \frac{d k^{\prime}}{k^{\prime}-k} \\
&+\frac{1}{2 \pi i} \int_{\Gamma_{+}} \frac{\underline{\varphi}\left(x, k^{\prime}\right)}{e^{i k^{\prime} \alpha} a\left(k^{\prime}\right)} \frac{d k^{\prime}}{k^{\prime}-k},
\end{aligned}
$$

where $\Gamma_{+}$is the infinite semicircle in the upper half plane (Fig.1). Using the expansion (53) and (45), it is straightforward to compute that the integral over $\Gamma_{+}$is simply $(1 / 2)\left(\xi(x) / \xi^{\prime}(x)\right)^{1 / 2}$.

Similarly,

$$
\begin{aligned}
-\underline{\psi}(x, k) & =\frac{1}{2 \pi i} \oint_{C_{-}} \underline{\psi}\left(x, k^{\prime}\right) \frac{d k^{\prime}}{k^{\prime}-k} \\
& =\frac{1}{2 \pi i} \int_{-\infty}^{\infty} \underline{\psi}\left(x, k^{\prime}\right) \frac{d k^{\prime}}{k^{\prime}-k}+\frac{1}{2 \pi i} \int_{\Gamma_{-}} \underline{\psi}\left(x, k^{\prime}\right) \frac{d k^{\prime}}{k^{\prime}-k},
\end{aligned}
$$

where $C_{-}$is the closed contour in the lower half plane, $\Gamma_{-}$is the infinite semicircle in the lower half plane (Fig.1). Due to (50), (52) the integral over $\Gamma_{-}$is $(1 / 2)\left(\xi(x) / \xi^{\prime}(x)\right)^{1 / 2}$.

Now, from (55) - (57) it follows that for $\operatorname{Im} k<0$,

$$
\begin{array}{r}
\underline{\psi}(x, k)=\left(\frac{\xi(x)}{\xi^{\prime}(x)}\right)^{1 / 2}+\int_{-\infty}^{\infty} \mathcal{R}\left(k^{\prime}\right) \underline{\bar{\psi}}\left(x, k^{\prime}\right)[\xi(x)]^{2 i k^{\prime}} \frac{d k^{\prime}}{k^{\prime}-k} \\
+\sum_{n=1}^{N} \frac{b_{n}[\xi(x)]^{-2 \kappa_{n}} \underline{\psi}\left(x,-i \kappa_{n}\right)}{a^{\prime}\left(i \kappa_{n}\right)\left(k-i \kappa_{n}\right)} .
\end{array}
$$

The expression (58), taken at $k=-i \kappa_{p}, p=1, \ldots, N$ gives

$$
\begin{array}{r}
\underline{\psi}\left(x,-i \kappa_{p}\right)=\left(\frac{\xi(x)}{\xi^{\prime}(x)}\right)^{1 / 2}+\int_{-\infty}^{\infty} \mathcal{R}\left(k^{\prime}\right) \underline{\underline{\psi}}\left(x, k^{\prime}\right)[\xi(x)]^{2 i k^{\prime}} \frac{d k^{\prime}}{k^{\prime}+i \kappa_{p}} \\
+i \sum_{n=1}^{N} \frac{b_{n}[\xi(x)]^{-2 \kappa_{n}} \underline{\psi}\left(x,-i \kappa_{n}\right)}{a^{\prime}\left(i \kappa_{n}\right)\left(\kappa_{p}+\kappa_{n}\right)} .
\end{array}
$$

The equations (58) - (59) represent a linear system, from which $\psi(x, k)$ (for real $k$ ) and $\psi\left(x,-i \kappa_{n}\right)$ can be expressed through $\xi$, which, indee $\bar{d}$ is yet an unknown function.

Let us now recall that $\lambda(-i / 2)=0$. Since $\psi(x, k)$ does not depend on $m$ for $\lambda=0$ and since $\psi(x, k)$ is defined by its asymptotics at $-\infty$, it follows that $\psi(x,-i / 2)=e^{-x / 2}$. Thus, for $k=-i / 2$, (58) gives 


$$
\begin{aligned}
& e^{-x / 2}[\xi(x)]^{1 / 2}=\left(\frac{\xi(x)}{\xi^{\prime}(x)}\right)^{1 / 2}+\int_{-\infty}^{\infty} \mathcal{R}\left(k^{\prime}\right) \underline{\bar{\psi}}\left(x, k^{\prime}\right)[\xi(x)]^{2 i k^{\prime}} \frac{d k^{\prime}}{k^{\prime}+i / 2} \\
& +i \sum_{n=1}^{N} \frac{b_{n}[\xi(x)]^{-2 \kappa_{n}} \underline{\psi}\left(x,-i \kappa_{n}\right)}{a^{\prime}\left(i \kappa_{n}\right)\left(\kappa_{n}+1 / 2\right)} .
\end{aligned}
$$

Since $\psi(x, k)$ and $\psi\left(x,-i \kappa_{n}\right)$ are known from (58) - (59), the equation (60) is a first order differential equation for $\xi$, which can be directly integrated to give $\xi(x)$. In other words, (58) - (60) represent a system of singular integral equations for $\underline{\psi}(x, k), \underline{\psi}\left(x,-i \kappa_{n}\right)$ and $\xi(x)$.

Since the time evolution of the scattering data is known (35), the dependence on $t$, i.e. $\xi(x, t)$ is also known, expressed by the scattering data. Thus the set $\mathcal{S}$, (28) uniquely determines the potential: from (35) one obtains

$$
m(x, t)=\omega\left[\left(\frac{\xi_{x}(x, t)}{\xi(x, t)}\right)^{2}-1\right]
$$

\section{Reflectionless potentials}

The inverse scattering is simplified in the important case of the so-called reflectionless potentials, when the scattering data is confined to the case $\mathcal{R}(k)=0$ for all real $k$. This class of potentials corresponds to the $N$ soliton solutions of the $\mathrm{CH}$ equation. In this case $b(k)=0$ and $|a(k)|=1$ (21) and from (46), (44) one can easily find that $i a^{\prime}\left(i \kappa_{p}\right)$ is real:

$$
i a^{\prime}\left(i \kappa_{p}\right)=\frac{1}{2 \kappa_{p}} e^{\alpha \kappa_{p}} \prod_{n \neq p} \frac{\kappa_{p}-\kappa_{n}}{\kappa_{p}+\kappa_{n}}
$$

where

$$
\alpha=\sum_{n=1}^{N} \ln \left(\frac{1+2 \kappa_{n}}{1-2 \kappa_{n}}\right)^{2} .
$$

Thus, $i a^{\prime}\left(i \kappa_{p}\right)$ has the same sign as $b_{n}$, (27) and therefore

$$
c_{n} \equiv \frac{b_{n}}{i a^{\prime}\left(i \kappa_{p}\right)}>0
$$

The time evolution of $c_{n}$ due to (35) is

$$
c_{n}(t)=c_{n}(0) \exp \left[\frac{4 \omega \kappa_{n}}{1-4 \kappa_{n}^{2}} t\right] .
$$

The equation (59) represents a linear system of equations for the quantities $\underline{\psi}\left(x,-i \kappa_{p}\right)$ : 
$\underline{\psi}\left(x,-i \kappa_{p}\right)+\sum_{n=1}^{N} \frac{c_{n}[\xi(x)]^{-2 \kappa_{n}}}{\kappa_{p}+\kappa_{n}} \underline{\psi}\left(x,-i \kappa_{n}\right)=\left(\frac{\xi(x)}{\xi^{\prime}(x)}\right)^{1 / 2}, \quad p=1, \ldots, N$

or

$$
\underline{\psi}\left(x,-i \kappa_{n}\right)=\left(\frac{\xi(x)}{\xi^{\prime}(x)}\right)^{1 / 2}\left[A^{-1} B\right]_{n}
$$

where

$$
A_{p n}[\xi, t] \equiv \delta_{p n}+\frac{c_{n}(t) \xi^{-2 \kappa_{n}}}{\kappa_{p}+\kappa_{n}}, \quad B \equiv[\underbrace{1,1, \ldots, 1}_{N}]^{t},
$$

i.e., finally

$$
\underline{\psi}\left(x,-i \kappa_{n}, t\right)=\left(\frac{\xi(x, t)}{\xi_{x}(x, t)}\right)^{1 / 2} \sum_{p=1}^{N} A_{n p}^{-1}[\xi, t], \quad n=1, \ldots, N .
$$

Now (60) gives (note that from (49) $\xi(-\infty, t)=0$ )

$$
x=X(\xi, t) \equiv \ln \int_{0}^{\xi}\left(1-\sum_{n, p} \frac{c_{n}(t) \underline{\xi}^{-2 \kappa_{n}}}{\kappa_{n}+1 / 2} A_{n p}^{-1}[\underline{\xi}, t]\right)^{-2} d \underline{\xi},
$$

the time evolution of $c_{n}$ is known (65). This represents an implicit relation from which $\xi$ can be expressed as a function of $x$ and $t$. Thus, in this case the scattering data uniquely determine $\xi=\xi(x, t)$ and therefore the potential $m(x, t)$ (61).

Using (70) and (61) we obtain the $N$-soliton solution. Indeed, for fixed coordinates $x_{0}, t_{0}$, since $x$ is a monotonically increasing function of $\xi$ there is a unique $\xi_{0}>0$ (which is treated as a parameter from now on), such that $x_{0}=X\left(\xi_{0}, t_{0}\right)$. Furthermore, we have

$$
\xi_{x}=X_{\xi}^{-1}(\xi, t)
$$

and from here and 61)

$$
m\left(x_{0}, t_{0}\right) \equiv m\left(X\left(\xi_{0}, t_{0}\right), t_{0}\right)=\omega\left[\left(\xi_{0} X_{\xi}\left(\xi_{0}, t_{0}\right)\right)^{-2}-1\right]
$$




$$
\begin{aligned}
u\left(x_{0}, t_{0}\right) & \equiv u\left(X\left(\xi_{0}, t_{0}\right), t_{0}\right) \\
& =\frac{1}{2} \int_{0}^{\infty} e^{-\left|X\left(\xi_{0}, t_{0}\right)-X\left(\underline{\xi}, t_{0}\right)\right|} m\left(X\left(\underline{\xi}, t_{0}\right), t_{0}\right) X_{\xi}\left(\underline{\xi}, t_{0}\right) d \underline{\xi} \\
& =\frac{\omega}{2} \int_{0}^{\infty} e^{-\left|x_{0}-X\left(\underline{\xi}, t_{0}\right)\right|} \underline{\xi}^{-2} X_{\xi}^{-1}\left(\underline{\xi}, t_{0}\right) d \underline{\xi}-\omega .
\end{aligned}
$$

Finally, the $N$-soliton solution is

$$
u(x, t)=\frac{\omega}{2} \int_{0}^{\infty} e^{-|x-X(\xi, t)|} \xi^{-2} X_{\xi}^{-1}(\xi, t) d \xi-\omega .
$$

Note that $X(\xi, t)$ is an explicitly defined function (70) in terms of the scattering data. Thus, the solution (73) does not depend on any additional parameter.

For example, for the one-soliton solution the function $X(\xi, t)$ is:

$$
X(\xi, t)=\ln \int_{0}^{\xi}\left[\frac{1+\frac{1}{2 \kappa_{1}} c_{1}(t) \underline{\xi}^{-2 \kappa_{1}}}{1+\left(\frac{1}{2 \kappa_{1}}-\frac{1}{\kappa_{1}+1 / 2}\right) c_{1}(t) \underline{\xi}^{-2 \kappa_{1}}}\right]^{2} d \underline{\xi} .
$$

Note that since $\frac{1}{2 \kappa_{1}}-\frac{1}{\kappa_{1}+1 / 2}>0[\mathrm{cf.}(\underline{26})]$ and $c_{1}(t)>0$ [cf. (64) $]$, both the nominator and the denominator in (74) are always positive and singularities do not appear.

\section{Conclusions}

In this paper the Inverse Scattering Method for the $\mathrm{CH}$ equation is outlined in the case when the solutions are confined to be functions in the Schwartz class, $\omega>0$ and $m(x, 0)+\omega>0$. The $N$-soliton solution is explicitly constructed. The inverse scattering based on a Liouville transform, which leads to a spectral problem of the standard form $-\Psi_{y y}+Q(y) \Psi=\mu \Psi$ (the Schrödinger equation) is developed in series of works 9, 26, 31, 32. In this case $Q$ and $m$ are related through the Ermakov-Pinney ordinary differential equation. The construction of the soliton solutions based on the bilinear representation of the $\mathrm{CH}$ equation (Hirota's method) is presented in [36, 37, 38, see also 33 for a parametric representation of the $N$-soliton solution. The situation when the condition $m(x, 0)+\omega>0$ on the initial data does not hold is more complicated and requires separate analysis [28, 3]. If $m(x, 0)+\omega$ changes sign there are infinitely many positive eigenvalues accumulating at infinity, and singularities can appear in finite time in the form of wave breaking ( $\inf _{x \in \mathbf{R}}\left\{u_{x}\right\} \rightarrow-\infty$, while $u$ stays uniformly bounded), cf. 9, 10. The inverse scattering for multi-peakon solutions (for their existence we must 
have $\omega=0$ ) is reported in [1, 2]. For the periodic solutions see [7, 14, 21] (in this setting a scaling transform shows that there are no qualitative differences between the cases $\omega=0$ and $\omega \neq 0$ ). The traveling-wave solutions of the $\mathrm{CH}$ equation $(\omega=0)$ are classified in 39. The Darboux transform for the $\mathrm{CH}$ equation is obtained in [41. The construction of multi-soliton and multi-positon solutions using the Darboux/Bäcklund transform is presented in $[22,23]$.

\section{Acknowledgements}

A.C. acknowledges funding from the Science Foundation Ireland, Grant 04/BR6/M0042. V.S.G. acknowledges funding from the Bulgarian National Science Foundation, Grant 1410, R.I.I. acknowledges funding from the Irish Research Council for Science, Engineering and Technology. The authors are grateful to both referees for useful comments and suggestions.

\section{References}

[1] Beals, R., Sattinger, D. and Szmigielski, J.: Acoustic scattering and the extended Korteweg-de Vries hierarchy. Adv. Math. 140, 190-206 (1998)

[2] Beals, R., Sattinger, D. and Szmigielski, J.: Multi-peakons and a theorem of Stieltjes. Inv. Problems 15, L1-L4 (1999)

[3] Bennewitz, C.: On the spectral problem associated with the CamassaHolm equation. J. Nonlinear Math. Phys. 11, 422-434 (2004)

[4] Birkhoff, G. and Rota, G.-C.: Ordinary differential equations. Waltham: Blaisdell Publishing Company, 1969

[5] Camassa, R. and Holm, D.: An integrable shallow water equation with peaked solitons. Phys. Rev. Lett. 71, 1661-1664 (1993)

[6] Camassa, R., Holm, D. and Hyman, J.: A new integrable shallow water equation. Adv. Appl. Mech. 31, (1994)

[7] Constantin, A.: On the inverse spectral problem for the Camassa-Holm equation. J. Funct. Anal. 155, 352-363 (1998)

[8] Constantin, A.: Existence of permanent and breaking waves for a shallow water equation: a geometric approach. Ann. Inst. Fourier (Grenoble) 50, 321-362 (2000)

[9] Constantin, A.: On the scattering problem for the Camassa-Holm equation. Proc. R. Soc. Lond. A457, 953-970 (2001) 
[10] Constantin, A. and Escher, J.: Wave breaking for nonlinear nonlocal shallow water equations. Acta Mathematica 181, 229-243 (1998)

[11] Constantin, A. and Ivanov, R.: Poisson structure and Action-Angle variables for the Camassa-Holm equation, Lett. Math. Phys. 76, 93108 (2006); nlin.SI/0602049

[12] Constantin, A., Kappeler, T., Kolev, B. and Topalov, P.: On geodesic exponential maps of the Virasoro group, Preprint No 13-2004, Institute of Mathematics, University of Zurich (2004)

[13] Constantin, A. and Kolev, B.: Geodesic flow on the diffeomorphism group of the circle. Comment. Math. Helv. 78, 787-804 (2003)

[14] Constantin, A. and McKean, H.P.: A shallow water equation on the circle. Commun. Pure Appl. Math. 52, 949-982 (1999)

[15] Constantin, A. and Strauss, W.: Stability of peakons. Commun. Pure Appl. Math. 53, 603-610 (2000)

[16] Constantin, A. and Strauss, W.: Stability of a class of solitary waves in compressible elastic rods. Phys. Lett. A 270, 140-148 (2000)

[17] Constantin, A. and Strauss, W.: Stability of the Camassa-Holm solitons. J. Nonlin. Sci. 12, 415-422 (2002)

[18] Dai, H.-H.: Model equations for nonlinear dispersive waves in a compressible Mooney-Rivlin rod. Acta Mech. 127, 193-207 (1998)

[19] Fisher, M. and Shiff, J.: The Camassa Holm equation: conserved quantities and the initial value problem. Phys. Lett. A 259, 371-376 (1999)

[20] Fokas, A. and Fuchssteiner, B.: Symplectic structures, their Bäcklund transformation and hereditary symmetries. Physica D4, 47-66 (1981)

[21] Gesztesy, F. and Holden, H.: Soliton Equations and Their AlgebroGeometric Solutions, Volume I: (1+1)-Dimensional Continuous Models, Cambridge studies in advanced mathematics, volume 79. Cambridge: Cambridge University Press, 2003

[22] Hone, A.: The associated Camassa-Holm equation and the KdV equation. J. Phys. A: Math. Gen. 32, L307-L314 (1999)

[23] Ivanov, R.I.: Conformal Properties and Bäcklund Transform for the Associated Camassa-Holm Equation. Phys. Lett. A 345, 235-243 (2005); nlin.SI/0507005.

[24] Ivanov, R.I.: Extended Camassa-Holm hierarchy and conserved quantities. Zeitschrift für Naturforschung 61a, 133-138 (2006); nlin.SI/0601066 
[25] Johnson, R.S.: Camassa-Holm, Korteweg-de Vries and related models for water waves. J. Fluid. Mech. 457, 63-82 (2002)

[26] Johnson, R.S.: On solutions of the Camassa-Holm equation. Proc. Roy. Soc. Lond. A 459, 1687-1708 (2003)

[27] Johnson, R.S.: The Camassa-Holm equation for water waves moving over a shear flow. Fluid Dynamics Research 33, 97-111 (2003)

[28] Kaup, D.J.: Evolution of the scattering data of the Camassa-Holm equation for general initial data. Stud. Appl. Math. 117, 149-164 (2006)

[29] Lenells, J.: The scattering approach for the Camassa-Holm equation. J. Nonlin. Math. Phys. 9, 389-393 (2002)

[30] Lenells, J.: Conservation laws of the Camassa-Holm equation. J. Phys. A: Math. Gen. 38, 869-880 (2005)

[31] Li, Y. and Zhang, J.: The multiple-soliton solutions of the CamassaHolm equation. Proc. R. Soc. Lond. A 460, 2617-2627 (2004)

[32] Li, Y.: Some water wave equations and integrability. J. Nonlinear Math. Phys. 12 (Suppl. 1), 466-481 (2005)

[33] Matsuno, Y.: Parametric representation for the multisoliton solution of the Camassa-Holm equation. J. Phys. Soc. Japan 74, 1983-1987 (2005); nlin.SI/0504055

[34] Misiolek, G.: A shallow water equation as a geodesic flow on the BottVirasoro group. J. Geom. Phys. 24, 203-208 (1998)

[35] Novikov, S.P., Manakov, S.V., Pitaevsky, L.P. and Zakharov, V.E.: Theory of solitons: the inverse scattering method. New York: Plenum, 1984

[36] Parker, A.: On the Camassa-Holm equation and a direct method of solution I. Bilinear form and solitary waves. Proc. R. Soc. Lond. A 460, 2929-2957 (2004)

[37] Parker, A.: On the Camassa-Holm equation and a direct method of solution II. Soliton solutions. Proc. R. Soc. Lond. A 461, 3611-3632 (2005)

[38] Parker, A.: On the Camassa-Holm equation and a direct method of solution III. N-soliton solutions. Proc. R. Soc. Lond. A 461, 3893-3911 (2005)

[39] Parkes, E. and Vakhnenko, V: Explicit solutions of the Camassa-Holm equation. Chaos, Solitons and Fractals 26, 1309-1316 (2005) 
[40] Reyes, E.: Geometric integrability of the Camassa-Holm equation. Lett. Math. Phys. 59, 117-131 (2002)

[41] Schiff, J.: The Camassa-Holm equation: a loopgroup approach. Physica D 121, 24-43 (1998) 